\title{
Tabularia
}

\section{L'édition des chartes et des cartulaires dans le Nord de la France}

The editing of charters and cartularies in northern France

\section{Benoît-Michel Tock}

\section{(2) OpenEdition}

Journals

Édition électronique

URL : http://journals.openedition.org/tabularia/491

DOI : 10.4000/tabularia.491

ISSN : 1630-7364

Éditeur :

CRAHAM - Centre Michel de Boüard, Presses universitaires de Caen

Référence électronique

Benoît-Michel Tock, "L'édition des chartes et des cartulaires dans le Nord de la France », Tabularia [En ligne], Les cartulaires normands. Bilan et perspectives de recherche, mis en ligne le 22 décembre 2009, consulté le 30 avril 2019. URL : http://journals.openedition.org/tabularia/491; DOI : 10.4000/ tabularia.491 


\title{
L'édition des chartes et des cartulaires dans le Nord de la France
}

\section{The editing of charters and cartularies in northern France}

\author{
Benoit-Michel Tock \\ Université de Strasbourg \\ EA 3400, Équipe de recherche en sciences historiques \\ btock@unistra.fr
}

Résumé:

Près de quatre siècles après les premières éditions de chartes dans le Nord de la France, le bilan qu'on peut dresser du travail de publication des actes est à la fois positif et réservé. La qualité des éditions de textes diplomatiques (comme de tous les textes médiévaux) s'est fortement améliorée, mais il est nécessaire d'inventer de nouvelles formes de publication de textes si on veut pouvoir étudier rapidement tous les textes conservés.

Mots-clés: chartes, édition, cartulaires, France, diplomatique.

\section{Abstract:}

Nearly four centuries after the first editions of Northern French charters, we can stress the success of the publication of charters, but also have some reservations about it. The quality of the editions of diplomatic texts, as of all medieval texts, did strongly increase, but it is now necessary to find new forms of publication if one want to study as soon as possible all preserved texts.

Keywords: charters, edition, cartularies, France, diplomatics.

Plusieurs raisons incitent à dresser aujourd'hui un état de l'édition des textes diplomatiques: le développement de l'électronique évidemment, qui permet un renouvellement considérable des principes et des méthodes, mais également le fait qu'en France l'édition de textes sort, depuis vingt ou trente ans, de l'engourdissement où elle était plongée depuis le début du XXe siècle. D'autre part, des travaux souvent récents, sur les originaux, les pancartes, les cartulaires, et même l'archivistique ancienne permettent de mieux comprendre quel était, lors de sa conception comme lors de ce qu'on appelle joliment la vie du document, le statut de l'acte écrit. Enfin, plus qu'avant, des comparaisons régionales sont désormais plus facilement possibles. 


\section{L'édition des chartes dans le Nord de la France: un bilan}

Commençons par dresser, à grands traits, une histoire et un bilan de l'édition de chartes et de cartulaires dans le Nord de la France ${ }^{1}$.

L'histoire d'abord. Dans l'état actuel (et sans doute provisoire) de mes recherches, le premier éditeur de chartes de la région est Ferry de Locre (Ferreolus Locrius), auteur en 1616 d'un Chronicon belgicum ${ }^{2}$. Ferry lance ainsi le principe de la publication d'une chronique (en réalité pratiquement sous la forme annalistique) dont les notations sont enrichies de citations de textes. Il serait un peu imprudent d'en faire un lointain héritier de Folcuin de Saint-Bertin (qu'il connait et utilise cependant), parce que ce dernier limitait son horizon à son monastère tandis que Ferry l'étend à un Belgicum qui est en fait surtout l'Artois, on peut en revanche rattacher Ferry à une tradition incarnée par un Jacques de Guise, auteur au XIV ${ }^{\mathrm{e}}$ siècle des Annales historie illustrium principum Hanonie, qui cite des textes antérieurs et que Ferry, d'ailleurs, utilise aussi ${ }^{3}$. On peut aussi relever, parmi les prédécesseurs de Ferry, un personnage qu'il a sans doute connu, François de Bar, grand-prieur d'Anchin de 1599 à 1606, auteur d'une Flandria monastica manuscrite, mais initialement destinée à être publiée et dont certaines rubriques sont nourries de transcriptions de chartes ${ }^{4}$.

La deuxième étape est la publication de recueils d'actes. Le premier nom à citer ici est celui d'Aubert le Mire (Aubertus Miraeus). En 1624, il publie à la fois une sorte de chronologie de l'histoire belge (Rerum belgicarum annales) ${ }^{5}$

1. Et tout d'abord quelle est la justification de ce cadre géographique? Il ne faut pas perdre de vue que l'ensemble formé par ces deux départements n'a pas de consistance historique: au Moyen Âge, il correspond à des parties des comtés de Flandre (donc, de la France) et de Hainaut (relevant de l'Empire), auxquels s'ajoutent dans leur intégralité, ou presque, les comtés de Boulogne et de Saint-Pol; du point de vue ecclésiastique, le diocèse d'Arras y figure en entier, mais les diocèses de Cambrai, Thérouanne et Tournai s'étendent également dans l'actuelle Belgique. Au XVII ${ }^{e}$ siècle, c'est-à-dire au moment où, on le verra, naît l'édition de chartes, le futur Nord/Pas-de-Calais fait partie, avec ses prolongements actuellement belges, des Pays-Bas espagnols. Le XVIII ${ }^{e}$ siècle verra, après la conquête française, s'inscrire dans les mentalités la frontière actuelle, sans évidemment que les liens, culturels notamment, ne se relâchent, surtout chez les historiens puisqu'ils partagent un passé commun. C'est dire qu'il serait absurde ici de ne pas évoquer aussi ce qui concerne l'actuelle Belgique.

2. Locrius, Ferreolus, Chronicon belgicum, Arras, 1616, et les nombreux travaux d'André Duchesne, comme par exemple son Histoire généalogique de la maison de Béthune, Paris, La Rivière Guillaume de éd., 1639. Sur Ferry de Locre, voir la Biographie nationale publiée par l'Académie royale des sciences, des lettres et des Beaux-Arts de Belgique, t. 12, Bruxelles, 1892-1893, col. 307308. Jacques De Meyere (mort en 1552), avait préparé, en complément à son Compendium chronicorum Flandriae, Nuremberg, 1538, un «Recueil de chartes et de lettres» perdu: v. FrIs, Victor, «Note sur les œuvres historiques de Jacques De Meyere», Bulletin de la commission royale d'Histoire, 84, 1920, p. 245-303.

3. Éd. E. SACKUR dans MGH SS, t. 30, p. 78-334.

4. Sur François de Bar voir Gerzaguet, Jean-Pierre, Les chartes de l'abbaye d'Anchin (1079-1201), Turnhout, Brepols éd. (Artem), 2005, p. 14-15 et Tock, Benoît-Michel et MiLis, Ludo, Monumenta Arroasiensia, Turnhout, Brepols éd., 2000 (Corpus Christianorum. Continuatio Mediaevalis, 175), p. XLIII-XLV

5. Miraeus, Aubertus, Rerum belgicarum annales in quibus christiane religionis et variorum apud Belgas principatuum origines ex vetustis tabulis principumque diplomatibus haust explicantur, Bruxelles, [1624]. 
et (le lien entre les deux livres est évident, et d'ailleurs souligné par leur auteur) un recueil d'actes, le Codex donationum piarum ${ }^{6}$. Par la suite, Miraeus publiera plusieurs autres volumes d'éditions de chartes ${ }^{7}$, et l'ensemble de ses publications fera l'objet un siècle plus tard d'une nouvelle édition augmentée ${ }^{8}$.

On notera donc que ce qui aurait pu constituer une étape intermédiaire, la publication d'actes en pièces justificatives, apparaît en fait après la publication de recueils d'actes. On peut citer André du Chesne, historiographe du roi, qui publie en 1639 une histoire de la maison de Béthune (et, dans d'autres régions, de Châtillon, de Richelieu... $)^{9}$. Du Chesne, cependant, n'accorde pas de place particulière aux chartes, et si d'après leur titre les «Preuves de l'histoire de la maison de Béthune» sont «tirées des chartes de diverses églises et abbayes, des registres du Parlement et de plusieurs Thrésors publics et particuliers", en fait, elles accueillent aussi des extraits de chroniques.

Une troisième étape consiste en la publication de corpus supra-régionaux: ce sont là les grands érudits des XVII ${ }^{e}$ et XVIII ${ }^{e}$ siècles qui se distinguent, et on peut citer ici les noms de d'Achéry, Mabillon...

Vient ensuite la publication de corpus d'actes par fonds d'archives. Le premier volume spécifiquement constitué de l'édition d'un fonds d'archives est pour le Nord de la France l'édition des actes de l'abbaye d'Auchy publiée en 1788 par dom Bétencourt ${ }^{10}$. Ces publications par fonds d'archives, qu'on peut appeler publications monographiques, connurent ensuite un succès qui ne s'est pas démenti puisque des dizaines de volumes de ce genre ont été publiés depuis pour le Nord de la France.

Une variante en est la publication monographique centrée non plus sur les bénéficiaires, mais sur les auteurs. On pourrait citer différents exemples plus ou moins anciens, depuis l'ouvrage déjà noté de Du Chesne sur les seigneurs

6. Miraeus, Aubertus, Codex donationum piarum in quo testamenta, codicilli, litterae fundationum, donationum, immunitatum, privilegiorum et alia piae liberalitatis monumenta a pontificibus, imperatoribus, regibus, ducibus ac comitibus in favorem ecclesiarum praesertim Belgicarum edita continentur, Bruxelles, 1624 .

7. ID., Diplomata belgica, Bruxelles, 1627 ; ID., Donationes belgicae, Anvers, 1629 ; ID., Notitia ecclesiarum Belgii, Anvers, $1630 \ldots$

8. Miraeus, Aubertus et Foppens, Jean-François, Opera diplomatica et historica, 4 vol., Louvain puis Bruxelles, 1723-1748.

9. DU CHESNE, André, Histoire généalogique de la maison de Béthune, justifiée par chartes de diverses églises et abbayes, arrests du Parlement, titres particuliers, épitaphes, chroniques et autres bonnes preuves, Paris, 1639. Sur cet auteur, voir Roman D'Amat, Jean-Charles, in Dictionnaire de biographie française, Letouzey et ANÉ éd., 1965, t. 11, col. 1231-1232.

10. Dom BÉTencourt, Collection des chartes de l'abbaye d'Auchy-lez-Hesdin, O.S.B., dépendante immédiatement du siège épiscopal de Boulogne, olim Théroüenne, imprimé à vingt cinq exemplaires seulement, et lettre pour lettre, avec gravure de sceaux, depuis la fondation de cette abbaye en 1079 jusqu'à l'année 1300 , s.l., 1788, 416 p. La page de titre n'a pas été imprimée. Le titre ci-dessus est repris à l'exemplaire de la Bibliothèque de l'Institut de France (cote $4^{\circ} \mathrm{X} 401 \mathrm{~A}$; je remercie MM. Francis Rapp et Roland Recht, membres de l'Institut, de m'avoir permis d'accéder à cet ouvrage), où il a été écrit à la main. L'exemplaire ne compte pas non plus de préface ou de lettre dédicatoire. Sur dom Bétencourt, voir la notice de Prevost, Michel, in Dictionnaire de biographie française, t. 6 , col. 338 . 
de Béthune jusqu'à celui d'Henri Malo sur Renaud de Dammartin, comte de Boulogne ${ }^{11}$. Mais il s'agit là d'études d'une personne ou d'une maison, incluant tous les documents concernant ceux-ci. Les vrais corpus, exhaustifs et traités de manière critique, commencent avec la publication des actes des comtes de Flandre de 1070 à 1128 par Fernand Vercauteren en 1938, à moins qu'on ne s'autorise une petite excursion au sud de l'Authie et qu'on cite un volume qui le mérite d'ailleurs amplement, le recueil des actes des comtes de Pontieu par Clovis Brunel ${ }^{12}$.

Enfin, la dernière étape en date, la sixième donc, est celle des bases de données et de l'avènement de l'électronique. La première, même si elle n'est pas encore publiée (mais elle le sera bientôt), est celle des chartes originales antérieures à 1121 conservées en France, développée par l'Artem de Nancy ${ }^{13}$. D'ampleur nationale, elle ne s'intéresse qu'aux actes originaux, ou présumés tels, dont elle offre une transcription nouvelle: il s'agit donc bien de nouvelles éditions. Le Pas-de-Calais (compris ici comme département de conservation des originaux) y est assez peu représenté (14 actes), le Nord davantage (143 actes). La recherche des actes est facilitée par cette base de données, mais ce sont surtout les travaux sur le vocabulaire et les formules qui sont concernés ici.

Mais les progrès permis par l'informatique sont d'abord dus au souci, non de l'édition, mais du répertoire des actes (bien que les deux ne soient jamais dissociables).

Ceux-ci, on le sait, sont dispersés dans un très grand nombre de fonds d'archives, en ce qui concerne les originaux et les copies, et d'ouvrages imprimés et de revues, en ce qui concerne les éditions. L'accès à cette masse d'informations dispersées a toujours posé problème aux historiens. Dès le XVIII e siècle, des bases de données sont constituées sur papier pour tenter de regrouper et d'ordonner toute cette matière. On peut envisager sous cet angle l'extraordinaire travail du "Cabinet des Chartes», chargé, dans les dernières années du règne de Louis XV, de constituer à Paris un vaste dépôt de copies de chartes de tout le royaume ${ }^{14}$. Mais le Cabinet des Chartes, bien que son travail fût très largement externalisé auprès de la Congrégation de Saint-Maur, était essentiellement une entreprise publique, destinée à conforter le pouvoir royal en France et à l'étranger.

Une autre entreprise datant de la même période, celle de la Table chronologique des diplômes, chartes, titres et actes imprimés concernant l'histoire de France, était en partie différente. Le but de de Bréquigny ${ }^{15}$ était de dresser un répertoire des

11. Malo, Henri, Un grand feudataire: Renaud de Dammartin et la coalition de Bouvines. Contribution à l'étude du règne de Philippe Auguste, H. Champion éd., Paris, 1898.

12. Vercauteren, Fernand, Actes des comtes de Flandre (1071-1128), Palais des Académies éd., Bruxelles, 1938. Brunel, Clovis, Recueil des actes des comtes de Pontieu, Imprimerie nationale, Paris, 1930.

13. Tock, Benoît-Michel, dir., La diplomatique française du Haut Moyen Âge. Inventaire des chartes originales antérieures à 1121 conservées en France, 2 vol., Brepols éd., Turnhout, 2001. Cette base de données sera consultable sur internet dès le début 2010.

14. Charmes, Xavier, Le comité des travaux historiques et scientifiques (Histoire et documents), 3 vol., Imprimerie royale éd., Paris, 1886.

15. DE BRÉQUIGNY, Louis George, Table chronologique des diplômes, chartes, titres et actes imprimés concernant l'histoire de France, t. 1, Paris, Imprimerie nationale, 1769. 
actes français imprimés. Mais, comme il le soulignait dans sa préface, il agissait en lien étroit avec le Cabinet des Chartes, puisqu'il s'agissait de permettre aux copistes du Cabinet des Chartes de «s'assurer su les pièces qui s'offriroient à leurs yeux avoient déjà été publiées; et dans le cas où elles l'auroient été, dans quelles livres on pouvoir les trouver, afin de comparer l'édition avec l'original ${ }^{16}$.

L'entreprise de de Bréquigny était vouée à l'échec par le gigantisme même de son objet (il cite le chiffre de 12000 volumes dépouillés), confronté à la petitesse des moyens disponibles. Au XIX ${ }^{\mathrm{e}}$ siècle en Belgique la Commission royale d'Histoire confia à Alphonse Wauters une entreprise similaire, au titre d'ailleurs inspiré de l'œuvre de de Bréquigny ${ }^{17}$. A. Wauters, au travail il est vrai très imparfait, entreprit de publier des suppléments, des suppléments aux suppléments, et ainsi de suite. À ce prix, son travail se poursuivit pendant plus d'un siècle. Mais il devenait d'usage de plus en plus mal commode.

Dans les années 1980 la commission royale d'histoire a voulu mettre à jour ce répertoire, et a sagement considéré qu'elle devait le faire en utilisant l'informatique. Le résultat est un inventaire à peu près exhaustif des actes antérieurs à 1200, quel que soit leur mode de conservation, concernant une zone géographique surréaliste, et donc très belge, comprenant les principautés constituant l'actuel espace belge, mais dans leur extension maximale: le comté de Flandre, par exemple, inclut Boulogne, l'Artois et Saint-Pol. La fusion entre ce répertoire et les travaux du Dictionnaire du Latin Médiéval Belge a permis l'intégration non seulement des analyses, mais aussi du texte des actes édités; cette fois cependant ce sont les frontières de la Belgique actuelle qui ont fourni les limites au travail ${ }^{18}$. Les travaux sur le vocabulaire et les formules sont facilités par cette base de données, mais avec des limites: les textes peuvent être issus de copies ou d'éditions parfois tardives ou médiocres, et surtout tous les textes ne sont pas repris. En revanche, le caractère (à peu près) exhaustif de l'entreprise rend à la fois facile et sûre la recherche des actes.

La base de données «belges» s'arrête, on l'a vu, à l'actuelle frontière francobelge en ce qui concerne l'édition des actes. Il fallait donc que la France développât sa propre base de données. Mais s'il est possible, grâce à un considérable effort financier de la commission royale d'histoire et à l'efficacité du maître d'œuvre de cette base, Philippe Demonty, de couvrir toute la Belgique jusqu'en 1200 en quelques années, une telle perspective n'était pas possible en France, beaucoup plus vaste et où la proportion d'actes inédits est d'ailleurs, du moins dans de nombreuses régions, assez élevée. D’où la naissance, il y a quelques années, du projet «Chartae Galliae», qui vise à rendre accessible et interrogeable, sur internet, un très grand nombre de chartes françaises jusqu'à la fin du XIII ${ }^{\mathrm{e}}$ siècle. Pour

16. DE BRÉQUignY, Louis George, Table chronologique (v. n. 15), p. V.

17. WAUters, Alphonse, Table chronologique des chartes et diplômes imprimés relatifs à l'histoire de la Belgique, 11 vol., Bruxelles, Hayez éd., 1866-1871.

18. Thesaurus Diplomaticus, un CD-Rom, Turnhout, 1997. Voir aussi Demonty, Philippe, «Le Thesaurus Diplomaticus, un instrument de travail pour une nouvelle approche en diplomatique médiévale", in La diplomatique urbaine en Europe au Moyen Âge [Actes coll. Gand, 1998], Walter Prevenier et Thérèse de Hemptinne (éd.), Louvain - Apeldoorn, Garant, 2000, p. 123-132. 
arriver rapidement à ce résultat, ce sont les chartes déjà éditées qui sont reprises. Sans prétention d'exhaustivité (parce que la masse documentaire est énorme, et encore en très large partie inédite), cette base de données aidera la recherche des actes, mais plus encore le travail sur le vocabulaire et les formules.

\section{Perspectives}

Malgré des siècles d'érudition, il reste bien des choses à faire. En font témoignage quelques corpus récents: le pourcentage d'actes inédits s'élève à 46 pour les chartes de l'abbaye d'Anchin ${ }^{19}$, 52,9 pour les actes des évêques d'Arras ${ }^{20}, 76,2$ pour les actes de l'abbaye de Vaucelles (sous presse)! Quant aux actes déjà édités, ils demandent parfois instamment à faire l'objet d'un nouveau travail d'édition: les textes ne sont édités que partiellement, toutes les copies utiles, en cas de perte de l'original, n'ont pas été vues, les erreurs de transcription sont nombreuses, les identifications sont mal faites... Les éditions des actes de Saint-Vaast d'Arras ${ }^{21}$ ou de Saint-Bertin ${ }^{22}$, les deux plus grosses abbayes de la région, devraient impérativement être refaites, et il en va de même pour de nombreux autres fonds, $y$ compris parfois pour des éditions récentes!

Il en va de même d'ailleurs pour les éditions regroupant les actes d'un même auteur: actes des comtes, actes des évêques essentiellement, mais également actes seigneuriaux. Dans le Nord de la France la situation n'est pas trop mauvaise à cet égard: l'édition des actes des comtes de Flandre est en voie d'achèvement, du moins jusqu'à la fin du XII siècle $^{23}$, et les actes des comtes de Saint-Pol ont été publiés jusqu'à la fin du XIII ${ }^{e}$ siècle ${ }^{24}$. Restent tout de même les actes des comtes de Hainaut ${ }^{25}$ et de Boulogne, sans compter les "petits » comtes de Guînes ou d'Hesdin. Parmi les évêques, seuls ceux d'Arras ont fait l'objet d'une édition jusqu'à la fin du XII siècle; l'édition des actes des évêques de Cambrai étant terminée jusqu'en $1092^{26}$, en cours pour la période 1092-1130, prévue pour la période $1130-1167$; l'édition des actes des évêques de Thérouanne est en bonne voie

19. Gerzaguet, Jean-Pierre, Les chartes de l'abbaye d'Anchin (1079-1201), Turnhout (Artem), Brepols éd., 2005.

20. Tock, Benoît-Michel, Les chartes des évêques d'Arras (1093-1203), Paris, CTHS, 1991.

21. VAN DrIVAL, Eugène, Cartulaire de l'abbaye de Saint-Vaast d'Arras rédigé au XII siècle par Guiman, Arras, A. Courtin éd., 1875.

22. Guérard, Benjamin, Cartulaire de l'abbaye de Saint-Bertin, Paris, 1840. Haigneré, Daniel, Les chartes de Saint-Bertin, 4 vol., Saint-Omer, H. d'Homont, 1886-1899.

23. Vercauteren, Fernand, Actes des comtes de Flandre (1071-1128), Commission royale d'histoire, Bruxelles, 1938; De Hemptinne, Thérèse et Verhulst, Adriaan, De oorkonden der graven van Vlaanderen (juli 1128 - september 1191). II. Uitgave, 2 vol. parus [pour 1128-1177], Bruxelles, Palais des Académies, 1988-2001; Prevenier, Walter, De oorkonden der graven van Vlaanderen (1191aanvang 1206), 3 vol., Bruxelles, Palais des Académies, 1964-1971.

24. NIEUs, Jean-François, Les chartes des comtes de Saint-Pol aux XII ${ }^{e}$ et XIII ${ }^{e}$ siècles, Turnhout, Brépols, 2008 (Artem).

25. Dont beaucoup ont été publiés par Duvivier, Charles, Actes et documents anciens concernant la Belgique, nouvelle série, Bruxelles, Kiessling et Cie, P. Weissenbrush, 1903.

26. VAn Mingroot, Erik, Les chartes de Gérard I ${ }^{e r}$, Liébert et Gérard II, évêques de Cambrai et d'Arras, comtes du Cambrésis (1012-1092/93), Louvain, Leuven university press, 2005. 
d'achèvement aussi, tandis qu'on attend les actes des évêques de Noyon-Tournai jusqu'en 1146, Tournai ensuite. Pour les actes seigneuriaux, rien de comparable au travail de W. M. Newman sur les seigneurs de Nesle ${ }^{27}$.

Le bilan montre donc un contraste entre des éditions remarquables (éditions des actes des comtes de Flandre par W. Prevenier d'abord, Th. de Hemptinne et A. Verhulst ensuite), des éditions satisfaisantes (l'édition des actes du chapitre collégial de Lille, par Hautcoeur ${ }^{28}$, bien qu'ancienne, est tout à fait utilisable), des éditions à refaire; entre des actes inédits, des actes mal édités, des actes édités plusieurs fois dans des éditions satisfaisantes ou excellentes (par exemple les actes de Thierry ou Philippe d'Alsace, comtes de Flandre, pour l'abbaye d'Anchin).

Et si on peut se réjouir, comme je l'ai souligné d'entrée de jeu, de la multiplication des éditions, il n'en reste pas moins qu'au train où vont les choses et vu l'ampleur de la tâche les médiévistes du Nord de la France ne sont pas prêts à pouvoir disposer d'éditions de tous les actes qui les intéressent, même en se bornant à la fin du XII ${ }^{e}$ siècle.

C'est ici, à nouveau, que les bases de données peuvent être utiles. Si le travail d'édition des textes s'est ralenti au cours du XXe siècle, ce n'est pas seulement parce qu'il a été dévalorisé, c'est aussi parce qu'il s'est compliqué. À juste titre, d'ailleurs. Mais le résultat est qu'il n'est plus possible, comme le faisaient les érudits des XVIII ${ }^{e}$-XIX ${ }^{e}$ siècles, de publier rapidement de grandes quantités d'actes. Or ces éditions anciennes, même quand elles sont imparfaites, rendent maintenant encore de grands services. C'est pour cela que, avec quelques collègues, j'ai lancé un programme de "publication des actes inédits du Nord de la France». Il s'agit en fait de publier, sur internet, le texte de tous les actes inédits, jusqu'à la fin du XII siècle, reçus par des institutions situées dans les actuels départements du Nord et du Pas-de-Calais. Quelque 800 actes ont été recensés. Plus de 400 sont déjà versés dans la base de données en cours de constitution. Le travail avance même tellement bien que l'extension vers la Somme et l'Aisne est déjà commencée.

Il s'agit de simples transcriptions, sans identifications, sans critique (sauf la datation des actes non datés); et lorsqu'il n'y a plus d'original mais plusieurs copies, seule une d'entre elles a été prise en compte. Faut-il y voir un rejet des éditions critiques traditionnelles? Pas du tout. Plutôt un travail préparatoire, qui permettra aux chercheurs de déjà utiliser un texte en attendant, sans doute longtemps parfois, de vraies éditions. La meilleure preuve qu'il ne s'agit en rien de rejeter les éditions traditionnelles est que ce travail va aboutir, précisément, à des éditions critiques: les chartes de l'abbaye de Vaucelles devraient pouvoir être publiées très prochainement.

Une autre direction de travail est l'amélioration de certaines éditions anciennes: la collation systématique de l'édition du cartulaire du chapitre cathédral d'Arras par le comte de Loisne a déjà été faite (mais elle n'est pas encore publiée), celle

27. Newman, William M., Les seigneurs de Nesle en Picardie (XII ${ }^{e}-X I I I^{e}$ siècle). Leurs chartes et leur histoire, 2 vol., Paris, Picard, 1971.

28. Hautcoeur, Édouard, Cartulaire de l'église collégiale Saint-Pierre de Lille, 2 vol., Lille-Paris, A. Picard, 1894. 
du cartulaire du chapitre Saint-Barthélemy de Béthune par le même est en route ${ }^{29}$, celle du grand cartulaire de Saint-Bertin par l'abbé Haigneré est prévue.

\section{Problèmes de l'édition aujourd'hui}

L'édition de chartes, de nos jours, est confrontée à différents problèmes que je voudrais brièvement traiter ici.

Première question: l'édition doit-elle porter sur des cartulaires ou sur des recueils d'actes? On l'a vu, une pratique fréquente en matière d'édition de textes diplomatiques consiste en l'édition d'un cartulaire manuscrit, sans égard pour le reste de la tradition diplomatique. C'est ce qu'ont fait Guérard et Haigneré pour Saint-Bertin ${ }^{30}$, Van Drival pour Saint-Vaast d'Arras, de Loisne pour les chapitres d'Arras et de Béthune, Fossier pour le prieuré d'Hesdin ${ }^{31}$. On remarquera qu'il s'agit donc surtout d'une pratique ancienne, et, en partie du moins, qui concerne des fonds d'archives gros et complexes, mettant en œuvre de nombreux manuscrits différents. C'est aussi une solution de facilité, puisqu'il est plus simple d'éditer un manuscrit que plusieurs, surtout s'il s'agit de dizaines ou centaines d'originaux, dispersés dans un grand nombre de liasses et pas forcément bien inventoriés; l'accès à ces documents est donc, dans les dépôts d'archives, plus compliqué et surtout plus long ${ }^{32}$.

Mais l'édition d'un manuscrit-cartulaire conserve des partisans, attachés au respect du document dans lequel ils trouvent les chartes qu'ils veulent étudier. Faut-il donc éditer un cartulaire manuscrit, ou un recueil d'actes?

Pour répondre à cette question, il faut partir de l'origine, c'est-à-dire des chartes. Chacune d'entre elles constitue un texte sui generis, mis par écrit à un moment précis, suite à la rencontre entre différentes personnes dont les intentions et les intérêts se sont trouvés, de gré ou sous une contrainte judiciaire ou autre, être concordants.

Le cartulaire, lui, parce qu'il donne à celui qui le réalise l'occasion de sélectionner, classer et modifier les actes, est une réécriture et une réinterprétation de ces actes, faites pour répondre aux problèmes qui se posaient à l'époque du cartulariste.

En d'autres termes, l'édition de chartes met en avant l'essence même des actes (les raisons et modalités de leur conception), l'édition des cartulaires met en avant l'utilisation des actes à un moment donné. Il me parait assez normal de privilégier la première vision des choses. D'autant que l'édition d'actes se fait

29. DE Loisne, Auguste, Le cartulaire de Saint-Barthélemy de Béthune, Arras, H. d'Omont éd., 1895 .

30. Encore qu'Haigneré tienne compte de la publication de Guérard et donc d'un autre cartulaire manuscrit.

31. Fossier, Robert, Cartulaire-chronique du prieuré Saint-Georges d'Hesdin, Paris, éd. du CNRS, 1988.

32. C'est d'ailleurs pour la même raison que l'extension du programme de publication des actes inédits du Nord de la France vers la Picardie se fait de manière privilégiée par le biais de cartulaires. 
normalement dans l'ordre chronologique. Or une des caractéristiques, sinon la caractéristique essentielle de l'histoire est qu'elle travaille sur le temps; par voie de conséquence la chronologie la plus exacte possible des faits qu'elle étudie est un des impératifs essentiels de l'historien.

Enfin, il est rare qu'on ne dispose, pour toute tradition des actes, que d'un seul et unique cartulaire. Le plus souvent on dispose au moins de quelques originaux, de copies d'érudits, de plusieurs cartulaires... La focalisation sur un cartulaire, fût-il particulièrement important ou prestigieux, ne peut se faire qu'au détriment des autres états de la tradition.

Mais que faire quand on ne dispose, comme seul état de la tradition, que d'un seul et unique cartulaire? J'aurais tendance, même en ce cas, à malgré tout éditer les actes et non le cartulaire en tant que tel, c'est-à-dire à revenir à l'ordre chronologique. Mais si je privilégie cette option, c'est que, dans la région et à l'intérieur de la période sur lesquelles je travaille, la très grande majorité des chartes sont datées, de sorte que l'ordre chronologique dans lequel je place des chartes (ce que faisaient d'ailleurs plusieurs cartularistes de la région) doit être approximativement correct. Là où les chartes ne sont généralement pas datées, il est vrai que la reconstitution d'un ordre chronologique devient très hasardeuse. En ce cas, pourquoi pas l'ordre du cartulaire? Bien que cela ne résolve pas la question lorsque sont également conservés d'autres cartulaires, des originaux, des copies d'érudits...

On observe un autre développement récent des éditions de chartes critiques, faites selon les principes traditionnels, mais publiées de manière électronique et non plus sur papier. Elles sont encore peu nombreuses en diplomatique, et il n'y en a pas pour le Nord-Pas-de-Calais. J'avoue une grande réticence par rapport à cette formule, du moins quand elle n'est pas accompagnée d'une édition papier.

Le principal avantage de ces éditions électroniques, qui sont souvent de très grande qualité (voir l'exemple du cartulaire blanc de Saint-Denis, ou, en Normandie, l'édition des chroniques latines du Mont-Saint-Michel), est la souplesse de consultation qu'offre l'informatique. En ce sens, elles sont très utiles et trouvent parfaitement leur place dans le paysage de l'édition scientifique ${ }^{33}$.

Cependant, dès lors qu'elles n'existent que sur support électronique et pas sur papier, ces éditions ne répondent pas une autre exigence de l'édition critique: celle-ci doit être un travail de référence, sûr, stable et accessible avec sûreté. Le papier est peut-être plus durable, malgré sa fragilité, qu'une électronique dont nous ne connaissons pas l'avenir. En tout cas, l'absence de publication papier fait sur l'avenir de l'électronique un pari que je trouve beaucoup trop audacieux. Les risques en la matière sont d'ailleurs aussi bien techniques (quel avenir à long terme pour nos sites actuels?) que politiques et économiques (aurons-nous toujours les moyens d'entretenir ces sites? Surtout, quelle pérennité pour des sites internet, même institutionnels, lorsque leurs auteurs ne sont plus en fonction).

33. On avance aussi souvent un autre argument en leur faveur: le support électronique favoriserait, davantage que le papier, les révisions et corrections. Théoriquement vrai, cet argument est rarement vérifié dans les faits: la non actualisation des sites est une des plaies d'internet. 
L'expérience montre d'ailleurs déjà que ces risques ne sont pas imaginaires: il est déjà arrivé que des sites disparaissent suite au départ de leur auteur. Or les éditions de textes sont utilisées, le plus souvent, pendant des décennies, souvent même pendant des siècles: il est de la plus grande imprudence de les confier à un support instable.

Le support électronique me paraît surtout approprié, en ce qui concerne les textes diplomatiques, aux bases de données. À vrai dire, il leur est même indispensable. L'édition critique traditionnelle et la base de données sont complémentaires mais ont chacune leurs principes, leurs avantages, leurs modalités. Et, pour moi, leur support.

\section{Bibliographie}

École des Chartes Conseils pour l'édition des textes médiévaux, fascicule 2: Actes et documents d'archives, Paris, 2001.

Guyotjeannin, Olivier, Pycke, Jacques et Tock, Benoît-Michel, Diplomatique médiévale, $3^{e}$ éd., Turnhout, Brepols, (L'Atelier du Médiéviste, 2), 2006. 